\title{
A Novel Downlink Interference Management Mechanism for Two-Tier OFDMA Femtocell Networks
}

\author{
Gwo-Jong Yu and Hoai Nam Bui Khac
}

\begin{abstract}
In wireless broadband access networks, almost indoor environment encounters serious coverage problem due to non-line of sight transmission. To improve the coverage, femtocell has been introduced as a promising technology. However, the deployment of femtocells may introduce extra interference to macrocell base stations. An effective interference management mechanism is required to optimize the system performance. In this paper, a resource allocation algorithm is proposed to resolve the two-tier downlink interference problem. The proposed scheme combines fractional frequency reuse (FFR) in macrocell and a heuristic graph-based channel assignment algorithm in femtocell to reduce the interference problem. Simulation results reveal that the proposed approach significantly improves the system throughput of the two-tier OFDMA networks.
\end{abstract}

Index Terms-Femtocells, interference management, resource allocation, Frational Frequence Reuse (FFR).

\section{INTRODUCTION}

Recently, the telecommunications industry has experienced fantastic growth in mobile Internet traffic, which is expected to continue in the coming years. Based on the Cisco VNI, the overall mobile data traffic will grow to 11.2 exabyte per month by 2017 [1]. Such growth is referred to as the Mobile Data Explosion. Since $66 \%$ of voice and $90 \%$ of data traffics are generated indoors [2], optimizing system performance implies improving indoor coverage and capacity. Among new emerging wireless technologies, femtocell is a cost effective approach for operators nowadays. A network which includes both macro base station and femtocells is commonly referred as heterogeneous networks (HetNet).

Femtocells consists of femto base stations (FBS) which are small, short-range $(10 \sim 30 \mathrm{~m})$, and low-power cellular base station (BS) (10 100 $\mathrm{mW})$ and typically are designed for use in a home or small business. Femtocells operate in a licensed spectrum owned by the mobile operator and enable fixed mobile convergence (FMC) service. FBS connects to core network via broadband technologies such as digital subscriber line (DSL) or cable modem [3].

Besides the benefits that femtocell can bring on for both operators and subscribers, there are some challenges which need be solved. Among the challenges, interference management is a key issue for researchers and mobile operators, especially in case of high network density in heterogeneous networks in next few years. Due to the

Manuscript received October 1, 2014; revised March 25, 2016.

Gwo-Jong $\mathrm{Yu}$ and Hoai Nam Bui Khac are with the Department of Computer Science and Information Engineering, Aletheia University, New Taipei City, 251 Taiwan (e-mail: yugj@mail.au.edu.tw, hoainam.bk2012@gmail.com). interference problem in uplink and downlink is quite different, to simply the problem, the downlink interference problem in femtocell network is taken into account in this paper. Orthogonal frequency division multiple access (OFDMA) is a multicarrier transmission technology. Due to its highly spectral efficiency and low implementation cost, it has been adopted in many high rate wireless transmission standards (LTE, WiMax, and WiFi). This paper takes LTE as an example to investigate the interference problem in OFDMA-based femtocell networks [4].

In heterogeneous networks, there are two types of interferences. The first one is cross-tier interference which occurs by the network elements from different tiers. For example, the interference between macrocell evolved NodeB (eNB) and femtocell home evolved NodeB (HeNB) is cross-tier interference. The second one is co-tier interference which occurs by the network elements from the same tier. For example, the interference between two HeNB is co-tier interference. According to the involved network elements (sender/receiver), interference types (cross-tier interference/co-tier interference), and transmission directions (uplink/downlink), interference scenario in HetNet can be summarized as shown in Fig. 1 [5].

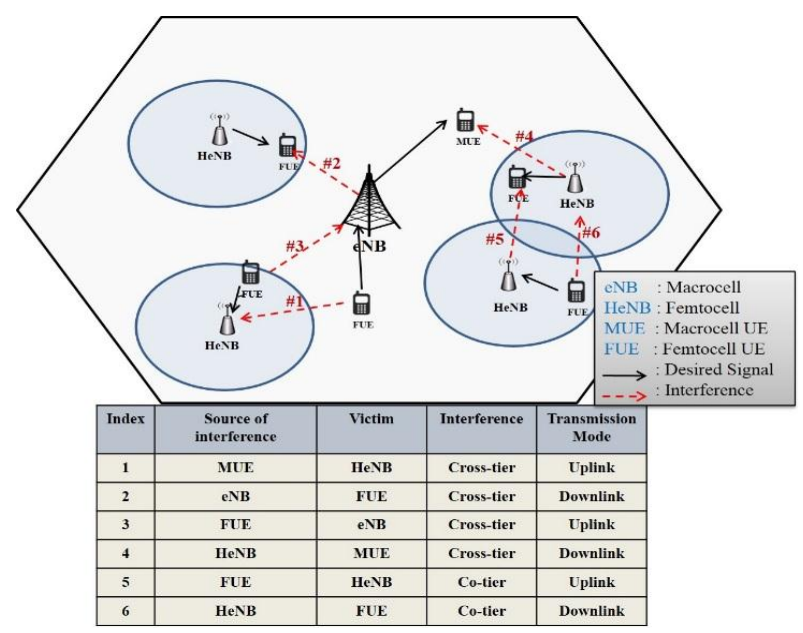

Fig. 1. Interference scenarios of each cell in heterogeneous network.

In this paper, an effective radio resource allocation mechanism is proposed to mitigate the interference in an OFDMA two-tier network. Since femtocells are managed by Femtocell Gateway (FGW) through the Internet, the FGW is designed to support resource assignment. The main contributions of this paper include: 1) A novel solution is proposed to solve the downlink interference problem in OFDMA-based femtocell network; 2) Both cross-tier and co-tier interferences are taken into consideration to achieve high system throughput for both macrocell and femtocell users; 3) The computational complexity of the proposed 
algorithm is not too complicated, so that the computation overhead is acceptable.

The remaining parts of this paper are organized as follows. In section II, related works about interference management is presented. Section III includes model problem formulation as well as the assumptions of the interference problem. In Section IV, the proposed algorithm FFR-GC is presented to solve the interference problem. Then, the simulation is performed to evaluate the performance of our algorithm in Section V. Finally, we conclude this paper in Section VI.

\section{RELATED WORK}

In order to reduce the interference, improve the link reliability, increase the capacity and enhance the system performance of femtocell networks, different kinds of interference management schemes have been investigated. In [3], Chandrasekhar and Andrews provided a good survey of femtocell technologies and provided guidelines about interference managements. Saquib et al. [5] summarized about the prominent issues and feasible approaches about femtocell interference problem as well as provided qualitative comparison among different schemes. In [6], Chandrasekhar and Andrews proposed to use sectorial antenna and multiple radio paths to enhance system capacity and to avoid uplink interference. Park et al. [7] used beam subset selection strategy and proposed an orthogonal random beam-forming based cross-tier interference reduction scheme in closed-access two-tier femtocell networks. Power control approaches are proposed as promising cost-effective methods [8]-[10].

In order to improve the resource allocation efficiency in OFDMA systems, the concept of frequency reuse have been introduced in [11]. In [12], Lopez-Perez et al. proposed two novel approaches for the self-organization of OFDMA femtocells, so that femtocells are able to dynamically sense the air interface and tune sub-channel allocation to reduce mutual interference. Tan et al. [13] used graph coloring algorithm and proposed a novel dynamic sub-band allocation technique (GC-DSA) to avoid downlink interference problem. GC-DSA exhibit high throughput in femtocell networks. However, this approach is not effective in case of large network with the high density of femtocell base stations. To deal with inefficient problem in high density networks, Zhang et al. [14] suggested to use cognitive approach to mitigate interference. The importance of cross-tier interference problem has also been discussed in literature. In [15], Lee et al. proposed to use fractional frequency reuse (FFR) in macrocell and assign unused sub-band for femtocell to minimize the interference between both cells. However, this approach only focuses in mitigating cross-tier interference between macrocell and femtocells. The co-tier interference among femtocells may also degrade system performance in dense networks.

\section{SYSTEM MODEL AND PROBLEM FORMULATION}

\section{A. System Model}

The objective of this paper proposed a novel mechanism to reduce interference in OFDMA Femtocell networks so that the throughput for the network can be improved. The network model that this paper investigates is illustrated in Fig. 2.

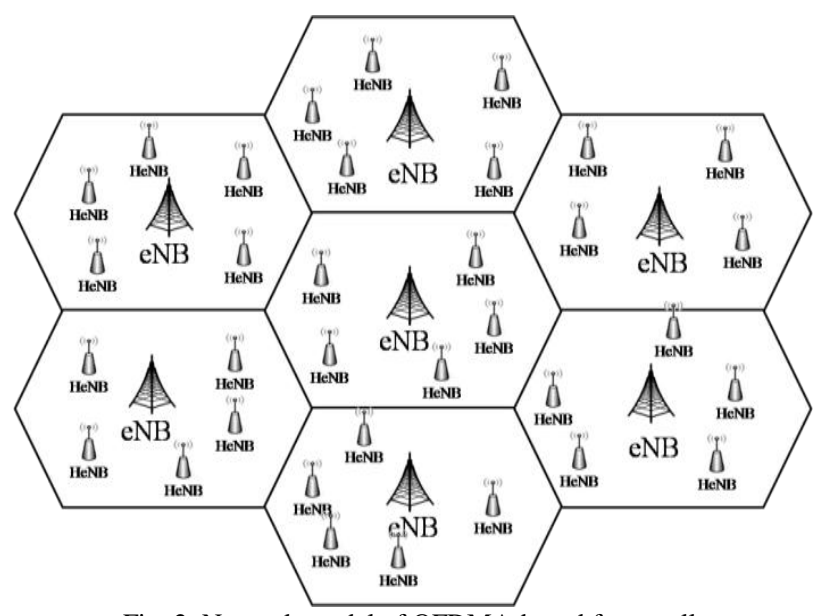

Fig. 2. Network model of OFDMA-based femtocell.

The target network is divided into 7 macrocells in which each macrocell was served by one eNB. A number of HeNB are deployed within a macrocell to enhance signal quality and system throughput. With the deployment of femtocells, if two network elements share the same radio resource, the interference in macrocell/femtocell pair or and femtocell/femtocell pair will occur. The objective of this paper is to investigate how to reduce both cross-tier interference and co-tier interference to enhance capacity of the network. The problem is formulated in the following.

\section{B. Problem Formulation}

Assume there are total $N_{M}$ macrocells in the network and each macrocell is served by an eNB. A number of femtocells are randomly distributed in entire network. The user equipment (UE) which is located outdoor and served by macrocell eNB is referred as macrocell user equipment (MUE) while the user equipment which is located indoor and served by femtocell HeNB is referred as femtocell user equipment (FUE). Assume the network is deployed in urban area, then the path loss model which described in [16] by 3GPP in LTE-advanced standard can be used to model the signal degradation. Let the network operates in $2 \mathrm{GHz}$, then the path loss (PL) between macrocell and MUE can be calculated as:

$$
P L_{\text {outdoor }}(d B)=15.3+37.6 \log _{10}(d)+\sigma^{2}
$$

In Eq. (1), $d$ is the distance (meter) between MUE $m$ and macrocell $M . \sigma^{2}$ is a factor which represents the outdoor log-normal shadowing (in $\mathrm{dB}$ ) and is characterized by the Gaussian distribution with zero mean and standard deviation [17]. Similarly, the path loss between FUE $f$ and femtocell $F$ can be calculated as:

$$
P L_{\text {indoor }}(d B)=38.46+20 \log _{10} d+L_{\text {walls }}
$$

The value of $L_{\text {wall }}$ depends on the distance between UE and BS (both macrocell and femtocell). The value, $L_{\text {wall }}$ equals $7 / 10 / 15(\mathrm{~dB})$ if $\mathrm{d}$ is within $(0-10 \mathrm{~m}) /(10 \mathrm{~m}-20 \mathrm{~m}) /(20 \mathrm{~m}-30$ $\mathrm{m})$, respectively. So, the channel gain $(G)$ for $i$-th UE can be 
calculated as:

$$
G=10^{-\frac{P L}{10}}
$$

In LTE standard, resource block (RB) is the minimum unit for radio resource allocation, the downlink signal to interference plus noise ratio (SINR) value on RB $a$ of MUE $m$ :

$$
\operatorname{SINR}_{m, M, a}=\frac{P_{M, a} G_{m, M, a}}{N_{0} \Delta f+\sum_{M^{\prime}} \beta_{m, a} P_{M^{\prime}, a} G_{m, M^{\prime}, a}+\sum_{F} \beta_{f, a} P_{F, a} G_{m, F, a}}
$$

In Eq. (4), $P_{M, a}$ and $P_{M^{\prime}, a}$ are transmit powers of serving macrocell and neighboring macrocell set $M^{\prime}$ on resource block $a . P_{F, a}$ is the set transmit power of femtocells which interfere to MUE $m$. $G$ is channel gain which can be computed by (3). $M^{\prime}$ and $F$ are sets of neighboring macrocells and femtocells, respectively. $\beta_{m, a}$ and $\beta_{f, a}$ denote the resource block $a$ is assigned for MUE $m$ and FUE $f \cdot \beta_{m(f), a}=1$ if resource block $a$ is assigned for MUF $m$ ( or FUE $f$ ), otherwise $\beta_{m(f), a}=0$. Similarly, SINR of FUEs $f$ is calculated:

$$
\operatorname{SINR}_{f, F, a}=\frac{P_{F, a} G_{m, F, a}}{N_{0} \Delta f+\sum_{F^{\prime}} \beta_{f, a} P_{F^{\prime}, a} G_{f, F^{\prime}, a}+\sum_{M} \beta_{m, a} P_{M, a} G_{f, M, a}}
$$

The capacity of MUE $m$ (or FUE $f$ ) on resource block $a$ can be given by (6):

$$
C_{m(f), a}=\Delta f * \log _{2}\left(1+\alpha \operatorname{SINR} R_{m(f), a}\right)
$$

In Eq. (6), $\alpha$ is a constant derived from bit error rate (BER) and can be defined $\alpha=-1.5 / \ln (5 B E R)$ with the setting of BER to $10^{-6}$.

Thus, the total throughput for each macrocell $M$ and femtocell $F$ can be calculated:

$$
T_{M(F)}=\sum_{m(f)=1}^{N_{m(f)}} \sum_{a=1}^{N_{a}} \beta_{m(f), a} C_{m(f), a}
$$

In generation, the objective of this paper is how to optimal the total throughput by allocating sub-bands for each femtocell after using FFR to assign sub-band for MUEs. Thus, we can formulate the problem of allocating sub-bands to femtocell as follows:

$$
\underset{\beta_{m, a} ;}{\max } \beta_{f, a}\left(\sum_{M=1}^{N_{M}}\left(\sum_{m=1}^{N_{m}} \sum_{a=1}^{N_{a}} \beta_{m, a} C_{m, a}+\sum_{F=1}^{N_{F}} \sum_{f=1}^{N_{f}} \sum_{a=1}^{N_{a}} \beta_{f, a} C_{f, a}\right)\right)
$$

Subject to:

$$
\begin{aligned}
& \text { 1) } \beta_{\mathrm{m}, \mathrm{a}} \times \beta_{\mathrm{f}, \mathrm{a}}=0 \forall a \in N_{a} \\
& \text { 2) } \beta_{\mathrm{f}, \mathrm{a}} \times \beta_{\mathrm{f}, \mathrm{a}}=0 \text { if } d_{f, f^{\prime}}<\mathrm{d}_{\text {threshold }} \\
& \text { 3) } \operatorname{SIN} R_{a, m}>\mathrm{SINR}_{\text {threshold }}
\end{aligned}
$$

In Eq. (8), $N_{M}$ is the total number of macrocells in entire networks and $N_{F}$ is the total number of femtocells within each macrocell coverage area. $N_{m}$ and $N_{f}$ are total number of MUEs and FUEs in each macrocell and femtocell, respectively. Subject 1 makes sure that there is no channel that assign for both MUE and FUE, and subject 2 dedicates that there is no channel assign for both femtocells which near to each other. In this way, the resource allocation problem becomes calculating the optimal parameter set $\beta_{f, a}$, such that the total throughput in Eq. (8) can be maximized. The proposed resource allocation mechanism is presented in next section.

\section{PROPOSED METHOD}

In this section, a fractional frequency reuse-based graph connectivity (FFR-GC) mechanism is proposed to solve the interference problem. FFR-GC scheme can be partitioned into two phases. In phase 1, the frequency sub-bands for macrocell users are allocated based on FFR to reduce interference experienced by MUEs. Then, a graph-coloring based algorithm which reduce the co-tier interference among femtocells and cross-tier interference between macrocell and femtocells is adopted in phase 2. This resource assignment sequence is reasonable since the traffic demand of MUEs should be fulfill first, then the throughput of FUE can be enhanced.

\section{A. Phase 1: Macrocell Radio Resource Allocation}

The FFR-based sub-band allocation mechanism proposed by Assaad [18] is adopted to assign sub-bands in macrocell. Each macrocell is partitioned into center zone and three edge zones (FFR-3) as show in Fig. 3. It is investigated in previous works that if the number of sector is larger than 3 , there is no significant performance improvement [19]. The simulation results presented in [18] show that when $2 / 3$ available bandwidth are allocated to center zone and the remaining $1 / 3$ available bandwidth is allocated to edge zones then the overall performance is the best. The size of sub-bands and the assignment of sub-bands to sub-area in macrocell using FFR mechanism illustrated in Fig. 3. Four frequency sub-bands $(A, B, C, D)$ are allocated to sub-areas of macrocell coverage area $(C 1, C 2, C 3$ for center zone and $E 1, E 2, E 3$ for edge zones). The detail sub-band assignment is illustrated in Fig. 3(b). Using FFR method, the interference for MUEs which located in edge zones will be reduced. For example, MUEs in edge zone $E 1$ can be interfered by macrocell 4 and macrocell 5 as shown in Fig. 3(a). Moreover, according to the above FFR method, the cross-tier interference between macrocell and femtocell are also avoided in each cell.

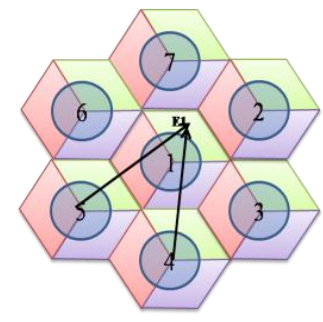

(a)

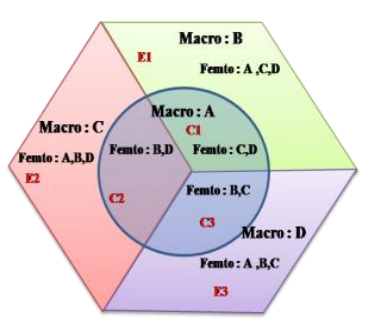

(b)
Fig. 3. Illustration of FFR macrocell frequency sub-bands assignment. (a) Sub-bands assignment in macrocell. (b) The sub-area layout and allocation for UEs using sectored-FFR.

As shown in Fig. 3(b), the MUEs and FUEs will be assigned by different sub-band in each sub-area. For example, 
in edge zone $E 1$, the sub-band $\mathrm{B}$ is assigned for macrocell, the remaining sub-bands, $\{A, C, D\}$, can be assigned for femtocell. In the center zone $C 1$, the femtocell can use sub-band $C$ and sub-band $D$ since sub-band $A$ has already been assigned to macrocell in center zone. The sub-band $B$ is assigned for macrocell edge zone $E 1$ area where is near to $C 1$ area. By this way, the cross-tier interference will be mitigated and the throughput over entire network will be enhanced.

\section{B. Phase 2: Frequency Sub-band Algorithm for Femtocell}

1) Sub-bands for femtocells

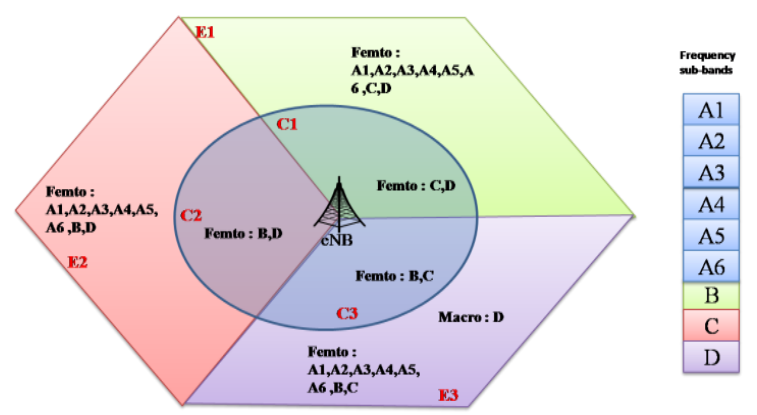

Fig. 4. The frequency allocation for femtocells.

As show in Section III, the resource allocation for femtocells is NP-Problem. Using the optimal algorithm to get the optimum resource assignment is not adequate since it requires the high complexity. Therefore, we propose the heuristic algorithm as a promising solution to solve with this problem. Thus, the propose algorithm may provide a non-optimal algorithm, but it give an efficient way, instead high complexity computation of the optimal algorithm.

To simplify the problem, we defined again the sub-bands for femtocell in each sub-area after using FFR for MUEs as shown in Fig. 3. Accordingly, the sub-band A will be divided into the six smaller sub-bands $\{\mathrm{A} 1, \mathrm{~A} 2, \mathrm{~A} 4, \mathrm{~A} 5, \mathrm{~A} 6\}$. Consequently, total bandwidth is partitioned into nine sub-bands equally (because sub-band $A$ equal $2 / 3$ total bandwidth).

The sub-bands which assigned for FUEs in each sub-area are shown in Fig. 4. Here, we can see the number of sub-bands that assigned for the FUEs in each sub-area of center zone only equal two while in each edge zone, there are eight sub-bands that are able to assign for UEs. This problem is feasible in practical system since almost the UEs in center zone will require data rates to macrocell base station to get stable signal instead using femtocell.

\section{2) Proposed algorithm for femtocell assignment}

The resource allocation problem can be translated into undirected graph problem where each femtocell is a vertex and the edge that connecting two vertices if they may interfere to each other. In this paper, we set the edge between two vertices if the distance between them smaller than $d_{\text {threshold }}$ which be defined up to the cell radius $R$ (cell coverage) of a femtocell base station:

$$
d_{\text {threshold }}=\underset{R(m)}{\arg }\left[\begin{array}{l}
\left(\sum_{f=1}^{N_{f}} \frac{B_{0}}{2} \times \log _{2}\left(1+\alpha \times \frac{\mathrm{P}_{\mathrm{F} 1} \mathrm{~g}_{\mathrm{F} 1 \mathrm{f}}}{\mathrm{N}_{0} \Delta \mathrm{f}}\right)=\right. \\
\left(\sum_{f=1}^{N_{f}} B_{0} \times \log _{2}\left(1+\alpha \times \frac{\mathrm{P}_{\mathrm{F} 1} \mathrm{~g}_{\mathrm{F} 1, \mathrm{f}}}{\left.\mathrm{N}_{0} \Delta \mathrm{f}+\mathrm{P}_{\mathrm{F} 2} \mathrm{~g}_{\mathrm{F} 2, \mathrm{f}}\right)}\right)\right.
\end{array}\right]
$$

In Eq. 9, $B_{0}$ is total bandwidth for two femtocells $F_{1}, F_{2}$ which are neighboring femtocells. Thus, the allocation problem transform into graph problem based on modified the maximal independent set (MIS). As the observation that the MIS algorithm usually gives a good result for reducing interference due to the set of femtocells using the same sub-bands is an independent set which not share the same channel. Moreover, we use iteration MIS for each sub-bands, so femtocell are able to reuse more than one sub-bands so the throughput will be enhanced. The process of the algorithm is given as follows:

1) The first step of the algorithm is to transform the system mode into undirected graph $\mathrm{G}(\mathrm{V}, \mathrm{E})$ with $V$ is the set of femtocell and $E$ is the set of interferences.

2) In second step, with each sub-band $s(s \in \mathrm{S}$ is total sub-band)), we find a graph $\mathrm{G}_{\mathrm{s}}\left(\mathrm{V}_{\mathrm{s}}, \mathrm{E}_{\mathrm{s}}\right)\left(\mathrm{G}_{\mathrm{s}} \in \mathrm{G}\right)$ obtains all the femtocell that the sub-band $s$ is able to assigned. For example, in Fig. 4, the femtocells which located in sub-area $E 1$ can use sub-bands $\{\mathrm{A} 1, \mathrm{~A} 2, \mathrm{~A} 3, \mathrm{~A} 4$, A5,A6,C,D . This thing is supported by FGW since the femtocells are managed by FGW where contain all information of each femtocell such as the locations, the neighboring femtocells and the sub-bands that able to be assigned.

3) The third step is based on modified maximal independent set algorithm for graph $G_{s}$ to find a set $\mathrm{K}$ which including all the independent femtocells (no interference) which assigned by sub-band $s$ (detail in algorithm 1 ).

4) Final step is repeating from step 2 until the last element of the set of sub-band S.

\section{3) Practical operation algorithm}

Since all the femtocells in network are managed by FGW, so we can know all information of each femtocell i.e locations, sub-bands are able to assigned for each femtocell.

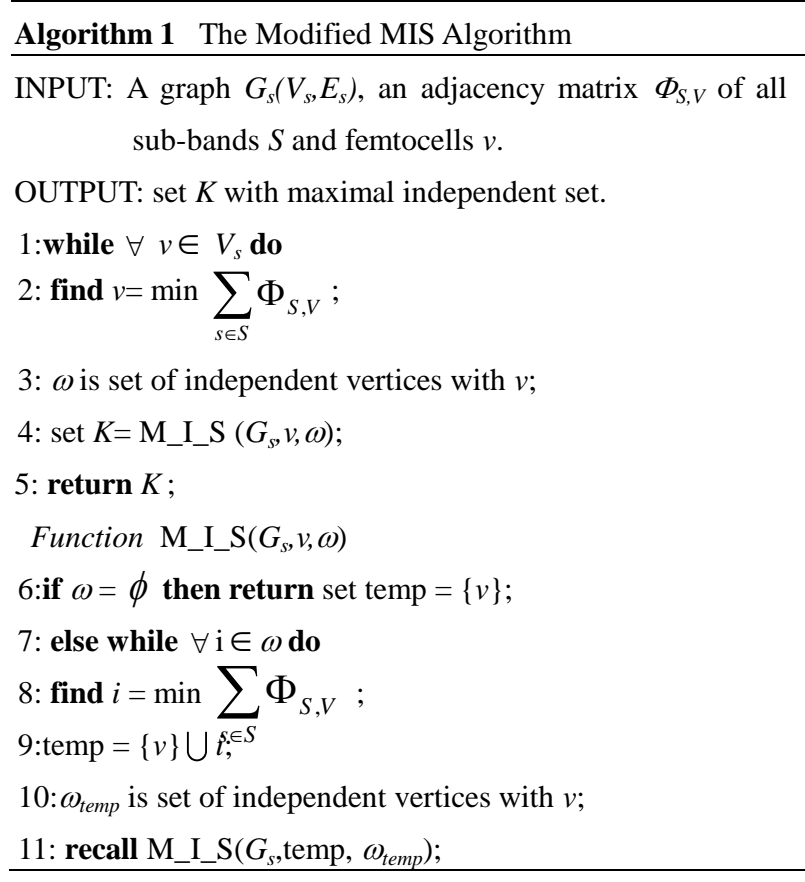

UEs in each sub-areas after allocating for macrocell UEs (Fig. 4). Therefore, for each sub-band, we can find all the femtocells in network which it is able to assign as example in 
Fig. 5(b,c,d). After defining sub-graphs for each sub-bands, the algorithm 1 presented which femtocell can use those sub-bands to avoid co-tier interference of femtocells as Fig. 5 (e).

The inputs are the sub-graph of each sub-bands and an adjacency matrix $\Phi$ which indicates the number of sub-bands assigning for each femtocell ( $\Phi_{s, v}=1$ if sub-band $s \in S$ assign for vertex $v \in V$ ). Typically, to make sure all femtocells can use frequency band (assuming each femtocell have the same data requirement) in each sub-graph, we will find femtocell $v$ is assigned least sub-bands ( $\min \Sigma \Phi_{S, V}$ ) with $S$ is total sub-band and beginning find the MIS from femtocell $v$ (function $M-I-S$ ) .

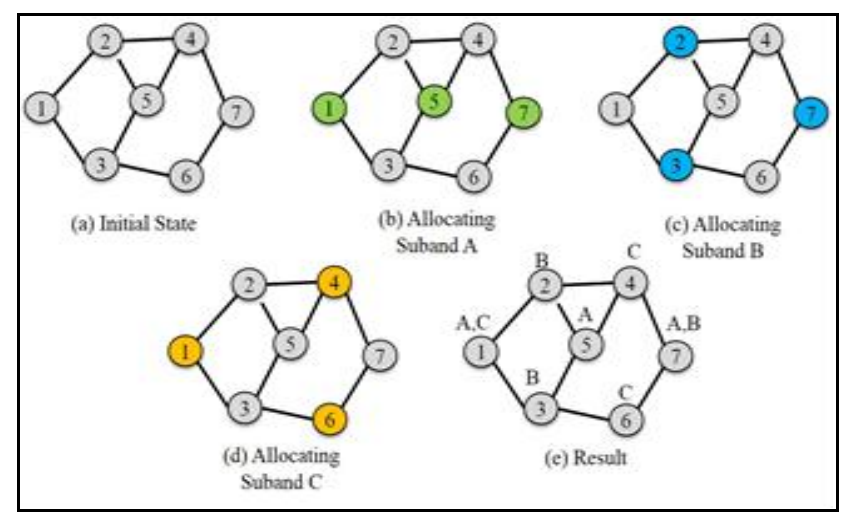

Fig. 5. An example of the modified MIS algorithm.

\section{PERformance EVAluation}

In this paper, we evaluate the proposed schemes in terms of throughput. We also concentrate on the performance of femtocell UEs and total UEs individually to get the fair evaluation about the proposed approach. We adopt the system parameters of 3GPP LTE, which are listed in Table I. The scenario is consisting of an area of 150 square meters. We vary the number of femtocells from 20 to 120 in one macrocell coverage in order to figure out the influence of variation of femtocell density. The macrocell UEs and femtocell UEs are randomly distributed in the overall network.

TABLE I: SIMULATION PARAMETERS

\begin{tabular}{|c|c|c|}
\hline Parameters & Macrocell & Femtocell \\
\hline Number of cell & 7 & $20-120 /$ cell \\
\hline Cell Coverage & $400 \mathrm{~m}$ & $30 \mathrm{~m}$ \\
\hline Transmit Power & $20 \mathrm{~W}$ & $20 \mathrm{~mW}$ \\
\hline Number of User per cell & 120 UEs & $120 \mathrm{UEs}$ \\
\hline Map Range per cell & \multicolumn{2}{|c|}{$150 \mathrm{~m} \times 150 \mathrm{~m}$} \\
\hline Channel Bandwidth & \multicolumn{2}{|c|}{$20 \mathrm{MHz}$} \\
\hline Carrier Frequency & \multicolumn{2}{|c|}{$2 \mathrm{GHz}$} \\
\hline Number of Resource Blocks & \multicolumn{2}{|c|}{$100 \mathrm{RBs}$} \\
\hline Spectrum of RB & \multicolumn{2}{|c|}{$180 \mathrm{kHz}$} \\
\hline White Noise & $-174 \mathrm{dBm}$ \\
\hline Lognormal Shadowing & \multicolumn{2}{|c|}{$10 \mathrm{~dB}$} \\
\hline
\end{tabular}

To show the benefits of the proposed approach, we compare the capacity of the proposed Fractional Frequency Reuse based on Graph Connectivity (FFR-GC) with other two schemes such as the Optimal-FFR scheme and Graph Coloring based Cognitive Spectrum Allocation (GC-CSA) scheme. For the optimal-FFR scheme in [18], they take an investigation in how to assign sub-bands for UEs using FFR method to get the best result. In this regards, we use the best result from this article to compare with our approach. The GC-CSA scheme was proposed in [13], in this article, they using graph coloring algorithm to assign sub-bands for femtocell.

To evaluate the proposed approach carefully, we firstly compare separately average throughput of macrocell UE (Fig. 6) and femtocell UE (Fig. 7). Then, Fig. 8 shows average throughput of UE in total network (both macrocell UEs and femtocell UEs).

Fig. 6 shows the average throughput of macrocell UEs, since using FFR method, cross-tier interference of both FFR-optimal approach and proposed approach (FFR-GC) will be reduced more effective than GC-CSA approach However, when the network gets high density of femtocells, the interference will be more complicated. The macrocell UEs in FFR Optimal approach will get more interference from neighboring femtocells. On the other hand, in our approach, we use graph algorithm to assign the sub-band for femtocell, the downlink interference from femtocell to macrocell UE will be reduce.

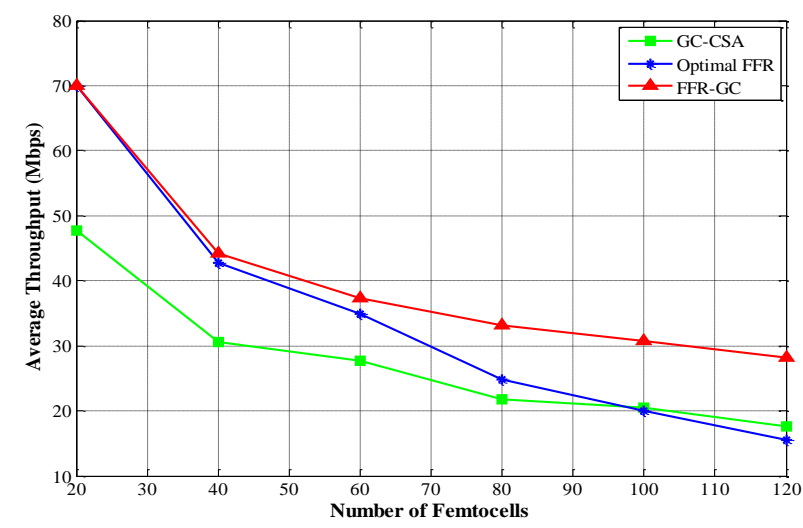

Fig. 6. Average throughput of macrocell UEs.

Fig. 7 shows the average throughput of femtocell UEs in each cell. We can see the domination of the proposed approach since it is able to get the higher SINR for each UE. The optimal-FFR only focus on using FFR for macrocell UEs and then reusing remaining sub-bands for femtocell UEs as much as possible, this thing bring on the high interference among femtocells. Meanwhile, the GC-CSA approach can make high results due to this approach use graph coloring to assign sub-bands for femtocell. Accordingly, the femtocells where nearby to each other will use different color to reduce the interference. But they do not consider interference from eNBs to FUEs so the throughput will be lower.

In Fig. 8, the average throughput of both MUEs and FUEs in total network is described. Using propose approach FFR-GC, The throughput of propose approach is always higher. Since it is not only mitigating cross-tier interference but also reducing co-tier interference. 


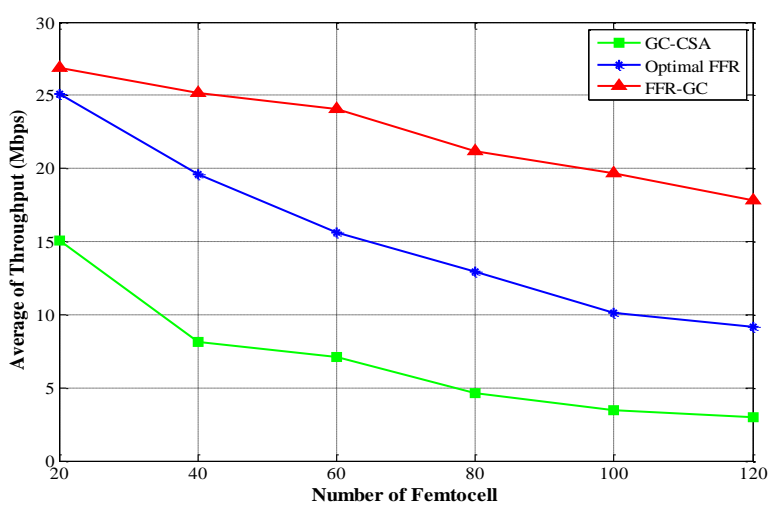

Fig. 7. Average throughput of femtocell UEs.

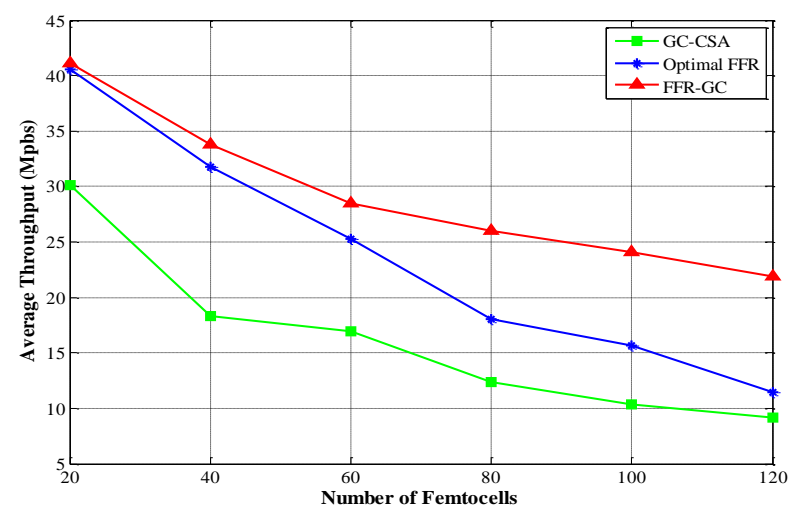

Fig. 8. Average throughput of UEs.

\section{CONCLUSION}

Recent years, femtocell is proposed as promising issues to provide higher throughput service with low cost in wireless cellular system. However, the interference in two-tier network in femtocell systems is a main challenge for the researchers as well as mobile operators. In this paper we propose an approach to deal with this problem. Firstly, we use FFR method to assign sub-bands for macrocell UEs to avoid cross-tier interference in each cell. Then, we propose a heuristic algorithm based on modified Maximal Independent Set algorithm to assign the sub-bands for Femtocell UEs to reduce co-tier interference as well as improve throughput. As results are shown in section $\mathrm{V}$, the proposed algorithm is not only reducing the interference in two-tier network but also improve throughput compare with previous approaches. Moreover, this approach also can be used in the large size of the network.

\section{ACKNOWLEDGMENT}

The authors would like to thanks the partial financial support from National Science Council in Taiwan with project number: NSC-103-2221-E-156-009.

\section{REFERENCES}

[1] Cisco White Paper, Cisco Visual Networking Index: Global Mobile Data Traffic Forecast 2012-2017, February 6, 2013.
[2] S. Carlaw, "IPR and the potential effect on femtocell markets," FemtocellsEurope, ABIresearch, 2008.

[3] V. Chandrasekhar and J. Andrews, "Femtocell networks: A survey," IEEE Commun. Mag., vol. 46, no. 9, pp. 59-67, Sept, 2008.

[4] 3GPP, “Ts 36.201 v8.1.0," Tech. Rep., April 2009.

[5] N. Saquib, E. Hossain, L. B. Le, and D. I. Kim, "Interference management in OFDMA femtocell networks: Issues and approaches," IEEE Wireless Commun. Mag., vol. 19, no. 3, pp. 86-95, June 2012.

[6] V. Chandrasekhar and J. G. Andrews, "Uplink capacity and interference avoidance for two-tier femtocell networks," IEEE Trans. Wireless Commun., vol. 8, no. 7, pp. 3498-3509, Jul. 2009.

[7] S. Park, W. Seo, Y. Kim, S. Lim, and D. Hong, "Beam subset selection strategy for interference reduction in two-tier femtocell networks," IEEE Trans. Wireless Commun., vol. 9, no. 11, pp. 3440-3449, Nov. 2009.

[8] M. S. Jin, S. Chae, and D. I. Kim, "Per cluster based opportunistic power control for heterogeneous networks," in Proc. IEEE VTC' 11-Spring, Buadapes, Hugary, May 2011.

[9] S. Gurauacharya, D. Niyato, E. Hossain, and D. I. Kim, "Hierarchical competition in femtocell-based cellular networks," in Proc. IEEE Globe-com' 10, Miami, FL, USA, 6-10 Dec., 2010.

[10] H. Jung, J. Han, and J. Lee, "Interference avoidance and resource allocation for OFDMA downlink of femtocells with distributed power control," IEICE Trans. Commun., vol. E93b, no. 4, pp. 1061-1064, Apr. 2010.

[11] D. Lopez-Perez, A. Valcarce, G. de la Roche, and J. Zhang, "OFDMA Femtocells: A roadmap on interference avoidance," IEEE Commu, Mag., vol. 47, pp. 41-48, Sept. 2009.

[12] D. Lopez-Perez, A. Ladanyi, A. Juttner, and J. Zhang, "OFDMA Femtocells: A-self-organizing approach for frequency assignment," in Proc. PIMRC, 2009, pp. 2202-2207.

[13] L. Tan, Z. Feng, W. Li, Z. Jing, and T. A. Gulliver, "Graph coloring based spectrum allocation for femtocell downlink interference mitigation," in Proc. IEEE Wireless Commun. Netw. Conf., Mar. 2011, pp. $1248-1252$.

[14] L. Zhang, L. Yang, and T. Yang, "Cognitive interference management for LTE-A femtocells with distributed carrier selection," in Proc. IEEE VTC', Sept. 2010, pp. 1-5.

[15] T. Lee, J. Yoon, S. Lee, and J. Shin, "Resource allocation analysis in OFDMA femtocells using fractional frequency reuse," in Proc. PIMRC, Sept. 2010, pp. 1224-1229.

[16] 3GPP, “Tr 36.814 v9.0.0,” Tech. Rep., March 2010.

[17] 3GPP, “ETSTTr 136.931 v9.0.0," Tech. Rep., May 2011

[18] M. Assaad, "Optimal fractional frequency reuse (FFR) in Multi-cellular OFDMA System," in Proc. IEEE VTC', Sept. 2008.

[19] D. Lee, C. Xu, U. Mayekar, and M. Mohile, "Frequency reuse factor vs. pathloss exponent and sectorization," IEEE MTT-S Symp. Tech. Wireless App. Digest, pp. 109-112, Feb. 1997.

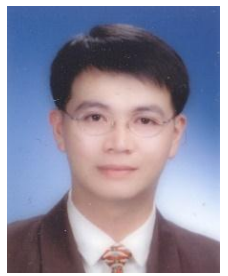

Gwo-Jong Yu received the B.S. degree in computer science from Christian University, Zhongli, Taiwan, in 1989, and the Ph.D. degree in computer science from the National Central University, Zhongli, Taiwan, in 2001.

Since August 2001, he was with the Faculty of Department of Computer Science and Information Engineering (CSIE), Aletheia University, New Taipei, Taiwan. He became a Professor at the Department of CSIE, Aletheia University, in 2011. His current research interests include wireless sensor networks, ad hoc networks, WiMAX, and LTE.

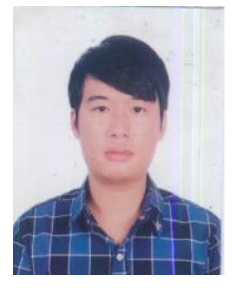

Hoai Nam Bui Khac was born in Quang Binh, Vietnam in 1988. He received the M.S. degree in the Department of Computer Science and Information Engineering (CSIE) in 2014 from Aletheia University, New Taipei, Taiwan. Currently, He is working in the Department of Information and Communications Technology in Quang Binh, Vietnam.

His research interests include radio resource management, interference management, and LTE. 